\title{
Social ideology and taxes in a differentiated candidates framework
}

\author{
By Stefan Krasa and Mattias Polborn
}

OnLine Appendix

\section{A1. Proof of propositions}

PROOF OF PROPOSITION 1:

First, suppose that $t_{D} \neq t_{R}$. Note that

$$
\begin{aligned}
& \frac{\partial m_{\delta}^{*}}{\partial t_{D}}=\frac{W_{D}^{\prime}\left(t_{D}\right)}{t_{D}-t_{R}}-\frac{W_{D}\left(t_{D}\right)-W_{R}\left(t_{R}\right)+\left(\delta_{R}^{2}-\delta_{D}^{2}\right)-2 \delta\left(\delta_{R}-\delta_{D}\right)}{\left(t_{D}-t_{R}\right)^{2}}, \\
& \frac{\partial m_{\delta}^{*}}{\partial t_{R}}=-\frac{W_{R}^{\prime}\left(t_{R}\right)}{t_{D}-t_{R}}+\frac{W_{D}\left(t_{D}\right)-W_{R}\left(t_{R}\right)+\left(\delta_{R}^{2}-\delta_{D}^{2}\right)-2 \delta\left(\delta_{R}-\delta_{D}\right)}{\left(t_{D}-t_{R}\right)^{2}} .
\end{aligned}
$$

Inserting these partial derivatives into the first order conditions and adding them implies that $W_{R}^{\prime}\left(t_{R}\right)=W_{D}^{\prime}\left(t_{D}\right)$. Furthermore, using the definition of $\bar{\delta}$ we get the right-hand side of (6).

We next show that $t_{D}=t_{R}$ cannot occur in equilibrium. To do this, we write the cutoff $\delta$ as a function of $m$ (rather than the other way around as we do in the main text). Solving for $\delta$ in (2), we get

$$
\delta_{m}^{*}=\frac{\left(W_{D}\left(t_{D}\right)-W_{R}\left(t_{R}\right)\right)-\left(t_{D}-t_{R}\right)}{2\left(\delta_{R}-\delta_{D}\right)}+\frac{\left(\delta_{R}+\delta_{D}\right)}{2 m}
$$

Denote by $J_{m}(\delta)$ the cumulative distribution of $\delta$ given $m$, and by $F(m)$ the marginal distribution of $m$. Then candidate $D$ 's vote share is given by

$$
V_{D}=\int J_{m}\left(\delta^{*}(m)\right) d F(m) .
$$

Taking the derivatives with respect to $t_{D}$ and $t_{R}$ provides the first order conditions. Adding the first order conditions again implies $W_{R}^{\prime}\left(t_{R}\right)=W_{D}^{\prime}\left(t_{D}\right)$. By assumption $W_{D}^{\prime}\left(t_{R}\right)>$ $W_{R}^{\prime}\left(t_{R}\right)$. Concavity of $W_{D}$ implies that $W_{D}^{\prime}(t)$ is decreasing in $t$. Thus, $W_{D}^{\prime}\left(t_{D}\right)=W_{R}^{\prime}\left(t_{R}\right)$ implies that $t_{D}>t_{R}$.

We next show that any interior solution to the first order conditions is unique. Let $t_{R}(t)=W_{R}^{\prime-1}\left(W_{D}^{\prime}(t)\right)$. Note that since $W_{R}^{\prime \prime}<0, W_{R}^{\prime}$ is strictly decreasing and hence the inverse $W_{R}^{\prime-1}$ exists. Now define

$$
h(t)=\frac{W_{D}(t)-W_{R}\left(t_{R}(t)\right)+\left(\delta_{R}^{2}-\delta_{D}^{2}\right)-2 \bar{\delta}\left(\delta_{R}-\delta_{D}\right)}{t-t_{R}(t)}-W_{D}^{\prime}(t) .
$$

Clearly, if $h\left(t^{*}\right)=0$ then $t_{D}=t^{*}$ and $t_{R}=t_{R}\left(t^{*}\right)$ satisfy the first order condition. Hence, 
the number of solutions of the first order conditions equals the number of zeros of function $h$ in $\left[\underline{t}_{D}, 1\right]$. We next show that $h^{\prime}(t)>0$ for any $t$ with $h(t)=0$.

$$
\begin{aligned}
h^{\prime}(t)= & \frac{W_{D}^{\prime}(t)-W_{R}^{\prime}\left(t_{R}(t)\right) t_{R}^{\prime}(t)}{t-t_{R}(t)} \\
& -\left(1-t_{R}^{\prime}(t)\right) \frac{W_{D}(t)-W_{R}\left(t_{R}(t)\right)+\left(\delta_{R}^{2}-\delta_{D}^{2}\right)-2 \bar{\delta}\left(\delta_{R}-\delta_{D}\right)}{\left(t-t_{R}(t)\right)^{2}}-W_{D}^{\prime \prime}(t) \\
= & \frac{\left.1-t_{R}^{\prime}(t)\right)}{t-t_{R}(t)}\left(W_{D}^{\prime}(t)-\frac{W_{D}(t)-W_{R}\left(t_{R}(t)\right)+\left(\delta_{R}^{2}-\delta_{D}^{2}\right)-2 \bar{\delta}\left(\delta_{R}-\delta_{D}\right)}{t-t_{R}(t)}\right)-W_{D}^{\prime \prime}(t) .
\end{aligned}
$$

Evaluated at $t=t^{*}$, the term in the parentheses in the second line is zero since $h\left(t^{*}\right)=0$, so that $h^{\prime}\left(t^{*}\right)=-W_{D}^{\prime \prime}\left(t^{*}\right)>0$. Intuitively, the result now follows since $h$ cannot have strictly positive derivatives if there is more than one $t$ with $h(t)=0$.

Formally, let $\underline{t}_{D}<t_{\min } \leq t_{\max }<1$ be the minimum and maximum, respectively over the set $\left\{t \mid h(t)=0, \underline{t}_{D}<t<1\right\}$. Let $\underline{t}$ be marginally smaller than $t_{\min }$ and $\bar{t}$ be marginally larger than $t_{\max }$. Then $h^{\prime}(\underline{t}), h(\bar{t})>0$ implies $h(\underline{t})<h(\bar{t})$. Let $\hat{h}$ be a the affine (linear) function on $\mathcal{T}=[\underline{t}, \bar{t}]$ defined by $\hat{h}(t)=(1 /(\bar{t}-t))[h(\underline{t})(\bar{t}-t)+h(\bar{t})(t-t)]$. Then the degree of $\hat{h}$ at any value $y$ of $\hat{h}$ is 1 because $\sum_{t \in \hat{h}^{-1}(y)} \operatorname{sign}\left(\hat{h}^{\prime}(t)\right)=\operatorname{sign}\left(\hat{h}^{\prime}\left(\hat{h}^{-1}(y)\right)\right)=$ $\operatorname{sign}((h(\bar{t})-h(\underline{t})) /(\bar{t}-\underline{t}))=1$. Since $\hat{t}$ is homotopic to $h$ via $H(x, t)=x h(t)+(1-x) \hat{h}(t)$ it follows that $h$ has degree 1. Let $n$ be the cardinality of the set $\mathcal{Z}=\{t \in \mathcal{T} \mid h(t)=0\}$. Since $h^{\prime}(t)>0$ for all $t \in \mathcal{Z}$ it follows that the degree of $h$ at 0 is $\sum_{t \in \mathcal{Z}} \operatorname{sign}\left(h^{\prime}(t)\right)=n$. Therefore $n=1$, i.e., there exists at most one solution to the first order conditions.

We now derive sufficient conditions for a local equilibrium. Taking the derivative of the first order condition of candidate $D$ in (4) with respect $t_{D}$ yields

$$
\int f_{\delta}^{\prime}\left(m_{\delta}^{*}\right)\left(\frac{\partial m_{\delta}^{*}}{\partial t_{D}}\right)^{2} d J(\delta)+\int f_{\delta}\left(m_{\delta}^{*}\right) \frac{\partial^{2} m_{\delta}^{*}}{\partial t_{D}^{2}} d J(\delta) .
$$

The first term is non-positive by the assumption that $f_{\delta}^{\prime} \leq 0 .{ }^{1}$ We now prove that the second summand is strictly negative. Note that

$$
\frac{\partial^{2} m_{\delta}^{*}}{\partial t_{D}^{2}}=\frac{W_{D}^{\prime \prime}\left(t_{D}\right)}{t_{D}-t_{R}}-\frac{2}{\left(t_{D}-t_{R}\right)} \frac{\partial m_{\delta}^{*}}{\partial t_{D}} .
$$

The first term in (A7) is strictly negative, and hence the integral that weighs this function with the joint density (i.e., $\left.f_{\delta}\left(m_{\delta}^{*}\right) d J(\delta)\right)$ is strictly negative. Integrating over the second summand in (A7), we get

$$
\int f_{\delta}\left(m_{\delta}^{*}\right) \frac{2}{\left(t_{D}-t_{R}\right)} \frac{\partial m_{\delta}^{*}}{\partial t_{D}} d J(\delta)=\frac{2}{\left(t_{D}-t_{R}\right)} \int f_{\delta}\left(m_{\delta}^{*}\right) \frac{\partial m_{\delta}^{*}}{\partial t_{D}} d J(\delta)=0,
$$

\footnotetext{
${ }^{1}$ Note that we only need the weaker condition that $f_{\delta}^{\prime}$ evaluated at the cutoff $m^{*}(\delta)$ is nonpositive for this conclusion.
} 
because the first order condition is satisfied. Thus, (A6) is strictly negative, i.e., the second order condition for maximization is satisfied.

The proof that the second order conditions are also satisfied for candidate $\mathrm{R}$ is analogous and omitted (note, though, that candidate $\mathrm{R}$ minimizes $V_{D}$, and therefore the second order condition is that the second derivative of $V_{D}$ with respect to $t_{R}$ is positive).

We next use Proposition 1 to derive necessary and sufficient conditions for the existence of solutions to the first order conditions.

PROPOSITION 1: Let $h$ be the function on $\left[t_{D}, 1\right]$ defined in (A5). Suppose that $W_{D}$ and $W_{R}$ are bounded from below and that the Inada conditions $W_{D}^{\prime}\left(\underline{t}_{D}\right)=W_{R}^{\prime}\left(\underline{t}_{R}\right)=\infty$ are satisfied. Then:

An interior solution of the first order condition exists if and only if $h(1)>0$.

\section{PROOF:}

The Inada condition implies that $\lim _{t \downarrow t_{d}} h(t)=-\infty$. If $h(1)>0$ then the intermediate value theorem implies that there exists a $t^{*}$ with $h\left(t^{*}\right)=0$, i.e., $t^{*}$ is a solution to the first order condition.

It remains to prove to no interior solution exists if $h(1) \leq 0$.

Suppose by way of contradiction that $h(1)=0$ and that an interior equilibrium $t^{*}$ exists. If $h(1)=0$ then the argument in the proof of Proposition 1 implies that $h^{\prime}(1)>0$. Therefore there exists $\bar{t}$ with $t^{*}<\bar{t}<1$ and $h(\bar{t})<0$. Since $h^{\prime}\left(t^{*}\right)>0$, there exists $\underline{t}$ with $t^{*}<\underline{t}<\bar{t}$ and $h(\underline{t})>0$. The intermediate value theorem implies that there exists $\hat{t} \bar{\epsilon}[\underline{t}, \bar{t}]$ with $h(\hat{t})=0$. Since $t^{*}<\hat{t}<1$ this contradicts the result on the uniqueness of interior solutions. Hence, no interior solution exists if $h(1)=0$.

Finally, suppose by way of contradiction that $h(1)<0$ and an interior solution $t^{*}$ to the first order condition exists. Then the argument in the proof of Proposition 1 implies that $h^{\prime}\left(t^{*}\right)>0$. Hence there exists $\hat{t}>t^{*}$ with $h\left(t^{*}\right)>0$. The intermediate value theorem implies that there exists a $t$ with $t^{*}<t<1$ with $h(t)=0$, i.e., a second solution to the first order condition exists, which contradicts the uniqueness result of Proposition 1.

PROOF OF PROPOSITION 2:

Equation (5) implies that $\bar{\delta}$ corresponds to the population average $E[\delta]$, and hence does not change when policies are changed (as long as the types are contested, i.e., $f\left(m_{\delta}^{*}\right)>0$ for all $\delta$ ).

Let $\psi=2 \bar{\delta}\left(\delta_{R}-\delta_{D}\right)-\left(\delta_{R}^{2}-\delta_{D}^{2}\right)$. Note that (6) can be written as

$$
\left(t_{D}-t_{R}+\psi\right) W_{R}^{\prime}\left(t_{R}\right)=W_{D}\left(t_{D}\right)-W_{R}\left(t_{R}\right) .
$$

Denote the derivatives of $t_{D}$ and $t_{R}$ with respect to $\psi$ by $t_{D}^{\prime}$ and $t_{R}^{\prime}$, respectively. Taking the implicit derivative in (A9) with respect to $\psi$ yields

$$
\left(t_{D}^{\prime}-t_{R}^{\prime}+1\right) W_{R}^{\prime}\left(t_{R}\right)+\left(t_{D}-t_{R}+\psi\right) W_{R}^{\prime \prime}\left(t_{R}\right) t_{R}^{\prime}=t_{D}^{\prime} W_{D}^{\prime}\left(t_{D}\right)-t_{R}^{\prime} W_{R}^{\prime}\left(t_{R}\right) .
$$

Since $W_{D}^{\prime}\left(t_{D}\right)=W_{R}^{\prime}\left(t_{R}\right)$ because of $(6)$, we get $W_{R}^{\prime}\left(t_{R}\right)+\left(t_{D}-t_{R}+\psi\right) W_{R}^{\prime \prime}\left(t_{R}\right)=0$, which 
implies that

$$
\frac{\partial t_{R}}{\partial \psi}=-\frac{W_{R}^{\prime}\left(t_{R}\right)}{W_{R}^{\prime \prime}\left(t_{R}\right)\left(t_{D}-t_{R}+\psi\right)}>0 .
$$

Thus, increasing $\psi=2 \bar{\delta}\left(\delta_{R}-\delta_{D}\right)-\left(\delta_{R}^{2}-\delta_{D}^{2}\right)$ increases $t_{R}$. It follows immediately from the first equality in (6) that increasing $\psi$ also increases $t_{D}$.

Now consider how $\psi$ changes as $\delta_{D}$ and $\delta_{R}$ change as stipulated in the proposition. The first claim follows because $\partial \psi / \partial \delta_{R}=2\left(\bar{\delta}-\delta_{R}\right)<0$ and $\partial \psi / \partial \delta_{D}=2\left(\delta_{D}-\bar{\delta}\right)<0$; note that, if the Democrat becomes more extreme or the Republican becomes more moderate, then the change in their respective positions is negative.

If both candidates become more extreme by $h$, then $\psi$ changes by $h\left(4 \bar{\delta}-\left(\delta_{R}+\delta_{D}\right)\right)$. Thus, $\psi$ increases (and taxes increase) if $\bar{\delta}>\left(\delta_{R}+\delta_{D}\right) / 2$, and $\psi$ decreases (and taxes decrease) if $\bar{\delta}<\left(\delta_{R}+\delta_{D}\right) / 2$.

\section{A2. Numerical example for differential production functions}

Here, we briefly present a numerical example illustrating our microfoundation for why Democratic and Republican candidates have different production functions. Let $g=$ $\sqrt{\bar{\gamma} t}-1$ be the production function. The wage in the public sector is $S=2$, and the intrinsic benefits for Democrats and Republicans are $z_{D}(t)=1.5-20(t-0.3)^{2}$ and $z_{R}=2-20(t-0.2)^{2}$, respectively. That is, Republican managers have a lower "ideal" tax rate than Democrats. Assume furthermore that $\bar{\gamma}\left(\gamma_{i}^{*}\right)=\gamma_{i}^{*}-0.02 .^{2}$

This example creates the two production functions displayed in Figure A1. Note that these two functions have the properties that we assume directly in the main text. The Republican has an advantage for low levels of taxation, while the Democrat has a higher marginal product and is eventually better than the Republican in terms of public good production for sufficiently high levels of taxation.

\section{A3. Empirical analysis}

We now present a short empirical analysis that shows the following two results for U.S. presidential elections from 1972 to 2008. First, voter behavior in these elections is consistent with the behavior depicted in Figure 1, in the sense that given any level of income, socially conservative voters are more likely to vote Republican than social liberals, and given any cultural position, the propensity to vote Republican increases in income. Second, the slope of the cutoff line in Figure 1 appears to have increased over time, in the sense that voter separation has become stronger with respect to cultural preferences, and more diluted with respect to income.

We consider the NES question on abortion (VCF0837/0838) as a measure for the respondent's cultural position; answer 1 that abortion should never be permitted is identi-

\footnotetext{
${ }^{2}$ Remember that candidate $i$ can only hire managers whose productivity is below $\gamma_{i}^{*}$, so the average productivity $\bar{\gamma}_{i}$ is lower than $\gamma_{i}^{*}$. This particular assumption (i.e., that $\bar{\gamma}\left(\gamma_{i}^{*}\right)=\gamma_{i}^{*}-0.02$, independent of the value of $\gamma_{i}^{*}$ can be derived from a uniform distribution of potential manager productivities, combined with the requirement to hire all applicants with productivity in $\left[\gamma_{i}^{*}-0.04, \gamma_{i}^{*}\right]$ in order to fill the available positions.
} 


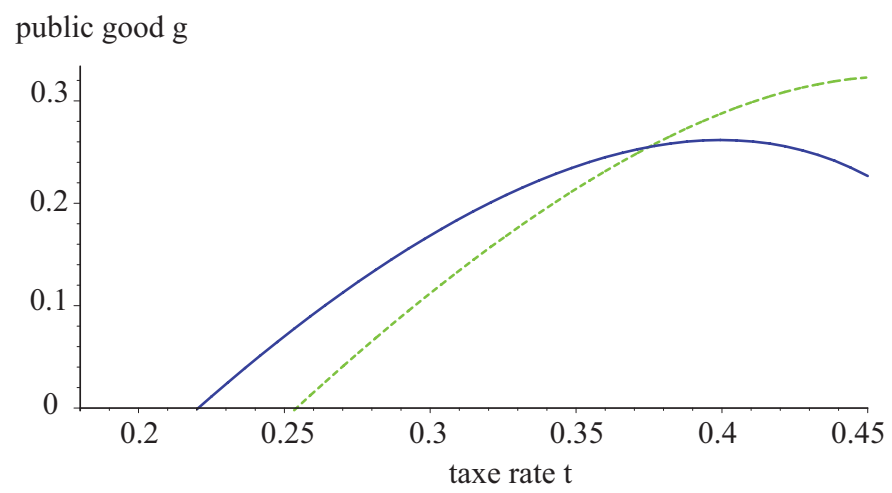

Figure A1. DifFERENTIATED PRODUCTION FUNCTIONS DERIVED VIA POLICY-MOTIVATED MANAGERS

fied as the conservative position, while response 4 that abortion should always be permitted is the most liberal response. Further, we identify the voters from the lowest third and the highest third of the income distribution, using question VCF0114, and denote them as "poor" and "rich."

Table A1 contains the probabilities that different voter types (in terms of their cultural and income positions) vote for the Democratic candidate in a Presidential election. To smooth out idiosyncratic variations between elections, we pool the data from the years 1972-1988 and those from 1988-2008.

Table A1-Fraction of Population Voting for Democrat

\begin{tabular}{|c|c|c|}
\hline Voter Type (Ideology, Income) & Vote Share 1972-1988 & Vote Share 1992-2008 \\
\hline (Liberal, Poor) & $63.0 \%$ & $77.9 \%$ \\
(Liberal, Rich) & $41.1 \%$ & $65.8 \%$ \\
(Conservative, Poor) & $59.0 \%$ & $29.8 \%$ \\
(Conservative, Rich) & $55.5 \%$ & $24.2 \%$ \\
\hline
\end{tabular}

We expect that, for both time periods, the following relationships must hold for the Democratic vote shares: (Liberal, Poor) $>$ (Liberal, Rich), (Conservative, Poor) $>($ Conservative, Rich), (Liberal, Poor) $>$ (Liberal, Rich), (Liberal, Poor) $>$ (Conservative, Poor). These four inequalities are indeed satisfied in Table A1. Moreover, except for the relationship that (Liberal, Poor) $>$ (Conservative, Poor) for 1972-1988, all relationships are significant at the $99 \%$ level. In addition, income became a worse predictor and ideology a better predictor for voting in the second period compared to the first one. This corresponds to a clockwise turn of the separating line in the right panel of Figure 1, resulting in the steeper separating line in the second half of the observation period.

We have argued above that the average cultural position of swing voters is to the right of the average cultural position of all voters. We now argue that this bias has increased as cultural preferences become more important. 
Consider again the case where $w(x)=\ln (x)$ and public goods are provided at constant marginal costs, i.e., $g_{D}(t)=a_{D}\left(t-b_{D}\right)$, and $g_{R}(t)=a_{R}\left(t-b_{R}\right)$. We have shown that $t_{D}-t_{R}=b_{D}-b_{R}$. Hence, the slope of the separating line (3) becomes steeper as the cultural difference between candidates $\delta_{R}-\delta_{D}$ increases. Suppose that income, $m$, follows an exponential distribution $\lambda e^{-\lambda m}$. $^{3}$ Let $k$ be the slope of the separating line, and $m_{0}$ the intercept. Suppose that $\delta$ is uniformly distributed on $[-1,1]$. For simplicity we further assume that the distributions of income and cultural preferences are independent. ${ }^{4}$ Then as long as $m_{0}-k \geq 0$, the average swing voter is given by

$$
\bar{\delta}=\frac{\int \lambda e^{-\lambda\left(k \delta+m_{0}\right)} \delta d \delta}{\int \lambda e^{-\lambda\left(k \delta+m_{0}\right)} d \delta}=\frac{\int e^{-\lambda k \delta} \delta d \delta}{\int e^{-\lambda k \delta} d \delta}=\frac{-e^{\lambda k}+\lambda k e^{\lambda k}+e^{-\lambda k}+\lambda k e^{-\lambda k}}{\lambda k\left(-e^{\lambda k}+e^{-\lambda k}\right)} .
$$

Note that if $k=0$, i.e., if cultural preferences do not matter and the separating lines is horizontal, then (A11) is zero, i.e., there is no difference between the average ideology of the swing voter and of the whole population. We next show that (A11) increases if $k$ is decreased.

$$
\frac{\partial \bar{\delta}}{\partial k}=\frac{2+4 \lambda^{2} k^{2}-\left(e^{2 \lambda k}+e^{-2 \lambda k}\right)}{\lambda k^{2}\left(e^{\lambda k}-e^{-\lambda k}\right)^{2}} .
$$

Note that

$$
e^{2 \lambda k}+e^{-2 \lambda k}=\sum_{n=0}^{\infty} \frac{(2 \lambda k)^{n}}{n !}+\sum_{n=0}^{\infty}(-1)^{n} \frac{(2 \lambda k)^{n}}{n !}=2 \sum_{n=0}^{\infty} \frac{(2 \lambda k)^{2 n}}{(2 n) !} \geq 2+2 \frac{(2 \lambda k)^{2}}{2 !},
$$

where the inequality is strict if $\lambda k \neq 0$. Hence, (A12) is strictly negative for $\lambda k \neq 0$.

In summary, if candidates' cultural positions diverge, then the separating line becomes steeper (more negative) which in turn means that the cultural preference of the average swing voter, $\bar{\delta}$, becomes more conservative relative to the population average.

Table A2 indicates, there has been a shift to a more liberal view on abortion, in the population, while the fraction of those who are completely opposed to abortion has stayed roughly the same at about $10 \%$. Thus, if the position of the average voter mattered, we should have seen a liberalization of the policies on abortions. In contrast, many states have moved in the opposite direction, imposing tighter restriction on abortion. This is compatible with our model, since the steeper slope of the separating line resulted in a right shift of $\bar{\delta}$ which can in turn outweigh the effect of the left-shift of the views of the population as a whole. In addition, politicians cater to swing voters who have more conservative positions, and the shift of preferences may have occurred primarily among non-swing voters.

\footnotetext{
${ }^{3}$ Note that this is a particularly simple form of an income distribution with a decreasing density.

${ }^{4}$ For example, the actual correlations between income and the abortion questions VCF0837 and VCF0838 in NES are -0.157 for $1972-1988$, and -0.108 for $1992-2008$ with confidence intervals of $[-0.182,-0.132]$ and $[-0.135,-0.081]$, respectively (if we recode the answers such that 1 is the most liberal, and 4 the most conservative position on this question). That is, wealthier people have slightly more liberal views on abortion.
} 
Table A2-Fraction of Respondents on Question VCF0837 and VCF0838 about abortion

\begin{tabular}{|c|c|c|c|c|}
\hline Years & Never Legal & Mostly Illegal & Mostly Legal & Always Legal \\
\hline $1972-1988$ & $10.3 \%$ & $39.8 \%$ & $18.8 \%$ & $31.1 \%$ \\
$1992-2008$ & $10.8 \%$ & $29.5 \%$ & $16.1 \%$ & $43.4 \%$ \\
\hline
\end{tabular}

Note the median answer to the abortion question has become more liberal, shifting from "abortion should be mostly illegal" to "abortion should be mostly legal." 5 Thus, in a Downsian model, where candidates can freely choose their position and are ex-ante identical, both would select a more liberal position on abortion, which contradicts the empirical evidence.

${ }^{5}$ For the exact wording of the question see the codebook of the NES. 